\title{
Interstitial pulmonary fibrosis associated connective tissue diseases - clinical and imaging aspects
}

\begin{abstract}
The lung is a common site of complications in systemic connective tissue diseases (CTD), and its involvement can present in several ways. Although it is generally thought that interstitial lung disease develops later on in CTD, it is often the initial presentation ("lung dominant" CTD). Interstitial lung disease (ILD) can be present in most types of CTD, including rheumatoid arthritis, systemic sclerosis, systemic lupus erythematosus, polymyositis or dermatomyositis, Sjögren's syndrome and mixed connective tissue disease. Despite similarities in clinical and pathologic presentation, the prognosis and treatment of CTD associated ILD (CTD-ILD) can differ greatly from that of other forms of ILD. Interstitial lung disease must be detected early in the course of collagen disorders by performing high-resolution computed tomography and pulmonary function tests. The pattern described on HRCT is predictive for treatment response and disease progression. Immunosuppression is the mainstay of treatment for ILD, although data from randomized controlled trials (RCTs) to support specific treatments are lacking. The management of patients with CTD-associated ILD is optimized by multidisciplinary collaboration.
\end{abstract}

Keywords: Interstitial lung disease (ILD), connective tissue diseases (CTD), high-resolution computed tomography (HRCT), pulmonary function tests

\section{INTRODUCTION}

The connective tissue diseases (CTDs) are a heterogeneous group of systemic disorders typically characterized by circulating autoantibodies and varying degrees of autoimmune mediated organ damage. Interstitial lung disease (ILD) is a common manifestation in CTDs such as rheumatoid arthritis (RA), systemic sclerosis (SSc), polymyositis/dermatomyositis (PM/DM), systemic lupus erythematosus (SLE), Sjogren's syndrome (SjS), and mixed CTD (MCTD). Pulmonary complications are common in CTD, have various presentations, and are associated with significant morbidity and mortality. Any of the compartments of the lung (i.e. airways, interstitium, vasculature and pleura) are at risk, and multicompartment involvement is a common finding in patients with CTD (1). The frequency of ILD in connective tissue diseases depends on the patient's selection and the modalities used to detect lung damage, but the prevalence is higher than initially con- sidered. A mutidisciplinary approach between pneumologist and rheumatologist identified that approximately $50 \%$ of patients classified as idiopathic interstitial pulmonary fibrosis (IIP) actually met criteria for ILD associated CTD (2).

The pathogenesis of autoimmune diseases is imprecisely clarified, but the role of autoimmunity in ILD in disorders such as rheumatoid arthritis, systemic sclerosis or systemic lupus erythematosus is well known at the moment (3). The primary trigger is considered acute lung injury that causes chronic interstitial inflammation followed by fibroblast activation and proliferation and finally pulmonary fibrosis with tissue destruction. Several histological subtypes are described in patients with rheumatic diseases, patterns identified also in idiopathic interstitial pulmonary fibrosis, the most common ones being: usual interstitial pneumonia (UIP); nonspecific interstitial pneumonia (NSIP); organizing pneumonia (OP) and lymphocytic interstitial pneumonia (LIP) (4). 
There is no consensus regarding ILD screening in patients with rheumatic diseases, but a careful history and a complete clinical examination, taking into account the potential pulmonary damage associated, is recommended in all patients. Specific tests should be performed in patients with suggestive signs and symptoms but also in those at high risk (systemic sclerosis) (5). Clinical manifestations may be nonspecific, including dyspnea with decreasing exercise tolerance, progressively accentuated within 2-6 months and dry cough. In addition, extrapulmonary manifestations (arthralgia, myalgia, cutaneous manifestations, lymphadenopathy, ocular, neurological or cardiac involvement) may be suggestive for diagnosis of CTD. On the clinical exam, bilateral dry basal crackels, hypocratic fingers or peripheral cyanosis may also occur. Pulmonary functional investigation is indispensable for diagnosis, disease progression assessment and prognosis. Respiratory function tests reveals restrictive ventilatory dysfunction with decreased total lung capacity, vital capacity and residual volume. Pathologic disturbance of the alveolarcapillary interface and also a decrease in gaseous transfer factor (DLCO - the diffusing capacity of the lung for carbon monoxide) is observed early compared to the decrease of vital capacity, being an important prognostic factor and monitoring the evolution of the disease. Also decrease the gas transfer constant $(\mathrm{kCO}=$ ratio DLCP/alveolar volume $)$ signifies alteration of diffusion by alveolo-capillary inflammation and not only by restrictive mechanism. Thoracic radiography, although an easily accessible and inexpensive method, is not sensitive enough to evaluate the incipient stages of ILD. The ready availability of high-resolution computed tomography (HRCT) has highlighted significant radiographic differences between diseases that have similar plain chest radiographic patterns. HRCT has the ability to better define the specific characteristics of lung parenchyma seen in each disease, increasing the chance of making a confident diagnosis. High resolution computed tomography (HRCT) is the gold standard in the diagnosis of interstitial lung fibrosis (6). HRCT assessment is complex and involves the description of the typical modifications of the ILD (ground glass, microreticulonodular infiltrates, distortion of the lung architecture and traction bronchiectasis, cystic pattern, called honeycombing) and the predominant histological type (UIP, NSPI, OP, LIP), therefore the investigation should be performed by an experienced radiologist.
A number of studies have shown that for idiopathic interstitial pulmonary fibrosis (IIP) the underlying histopathologic lung injury pattern determines prognosis: the fibrotic lung patterns (e.g., UIP and fibrotic-NSIP) are associated with a worse prognosis than more cellular patterns (e.g., cellular-NSIP, OP, and LIP). In contrast, but based on less robust data, it appears that for CTD-ILD the underlying histopathologic pattern does not convey similar prognostic significance. Rather, for CTD-ILD, the factors that are associated with a worse prognosis are older age, higher degree of dyspnea and worse pulmonary function. The only exception may be for RA as recent data suggest that RA-UIP may be associated with a worse prognosis than RA-NSIP $(7,8)$.

In the following we will present the association of interstitial lung fibrosis in various connective tissue diseases with some example of clinical cases.

\section{Rheumatoid arthritis and interstitial pulmonary fibrosis}

Rheumatoid arthritis is a systemic autoimmune disease characterized by an inflammatory and often destructive arthropathy that affects approximately $1 \%$ of the population. Extra-articular manifestations are associated with significant morbidity and increased mortality, and the lungs are frequently involved. The estimated prevalence of subclinical lung disease ranges from $19 \%$ to $58 \%$ (9). However, clinically significant lung disease occurs far less frequently. The strongest risk factor for the development of ILD in patients with RA is cigarette smoking. Other potential risk factors for the development of RA-ILD include male gender, high titer of rheumatoid factor (RF), anticyclic citrullinated peptide (anti-CCP) positivity and severe joint disease (10). Thoracic high-resolution computed tomography scans often demonstrate a combination of airways and parenchymal disease, and these findings may be subclinical in nature. Clinically significant ILD most commonly reveals patterns consistent with UIP (reticular opacities with microcysts, sometimes the appearance of ,honeycomb“ in the inferior and subpleural lobes) associated with an unfavorable prognosis or less frequently NSPI (11).

Unlike other CTDs, in which histopathologic pattern does not appear to impact survival, in RA, recent data from small series suggest that those with UIP do worse than those with other patterns. Indeed, in one study of RA-ILD, 5-year survival rates were 
noted to be better in those with an NSIP pattern compared to those with a UIP pattern (94\% vs 36\%) (12). The progression of pulmonary damage in these patients is approximately $50 \%$ in less than 1,5 years, the terapeutical decision must also take into account the histological type. Current treatment regimens are clinician dependent and typically involve chronic immunosuppression with corticosteroids along with corticosteroid sparing agents (e.g., azathioprine [AZA], cyclophosphamide [CYC] and mycophenolate mofetil [MMF]) (10).

We present the case of a 67-year-old (B.I.) patient diagnosed with seronegative rheumatoid arthritis in 2009 treated with methotrexate up to $10 \mathrm{mg} /$ week developing diffuse interstitial pneumopathy after approximately 6 years of treatment. The bronchoalveo- lar lavage reveals lymphocyte alveolitis (74.2\%), and computed tomography imaging shows interstitial changes of the reticulo-micronodular type, especially in lower lobes (Fig. 1). The quasi-complete resolution of the symptomatology, as well as the improvement in functional parameters after corticotherapy and discontinuation of methotrexate treatment suggested that the pulmonary involvement was associated mainly to immunosuppressive therapy.

Studies related to methotrexate-induced pneumopathy management have shown that progression to pulmonary fibrosis is low, especially if discontinuing treatment as quickly as possible, resolution of symptoms is common even without cortisone treatment; however, pulmonary toxicity to methotrexate may reach a mortality rate of up to $13 \%$ (13).

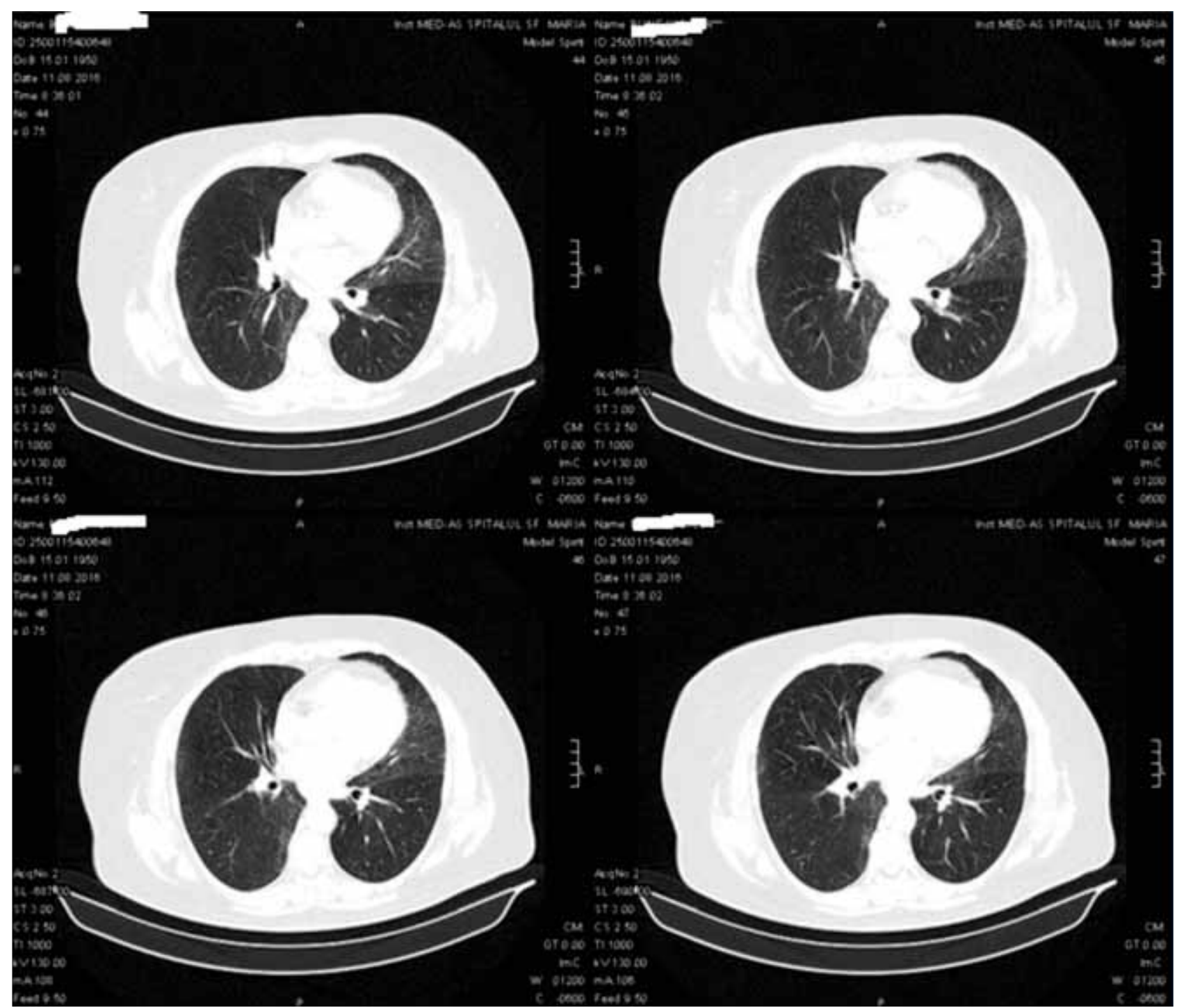

FIGURE 1. Reticulo-micronodular pattern in a patient with interstitial pneumopathy secondary to methotrexate administration 


\section{Systemic sclerosis and interstitial pulmonary fibrosis}

Lung pathology of systemic sclerosis (SSc) includes pulmonary hypertension and interstitial pulmonary fibrosis, both are important causes of death in these patients. When unselected patients are screened with HRCT, up to $90 \%$ will have some degree of ILD. Progressive SSc-ILD typically occurs within the first 5 to 7 years and is more common in patients with antitopoisomerase (anti-Scl-70) or nucleolar-staining ANA positivity and diffuse skin involvement (13). The HRCT most commonly reveals diffuse parenchymal ground glass infiltrates suggestive of the lung injury pattern of NSIP; coarse reticulation and honeycombing are less commonly seen, with the thickening of the interlobular septa and the preservation of the architecture, the affection being diffuse. Progress from the appearance of ground glass to reticular aspect with significant disorganization occurs in 2 out of 3 patients with SSc. It is considered extensive affection of $>20 \%$ of the lung surface, the situation being associated with increased risk of death (14). Recent data from controlled studies suggest that short-term treatment with CYC is associated with either modest or no improvement in forced vital capacity (FVC) compared with placebo, and these studies have dampened enthusiasm for treatment with this cytotoxic agent. Recent - though retrospective - data suggest that MMF may be a promising therapy for SSc-ILD as it appears to be well tolerated and associated with preservation of lung function (15).

We present the case of a 65 years old patient (C.A.) with a history of cervical cancer operated and iradiated, diagnosed in 2001 with CREST syndrome that develops after about 14 years of evolution of the disease interstitial lung fibrosis, with deterioration in functional parameters, decreased exercise tolerance with significant desaturation, requiring oxygen therapy at home. Computerized tomography (CT) scan reveals bilateral ground glass mostly basal (Fig. 2).

\section{Polymyositis/dermatomyositis and interstitial pulmonary fibrosis}

Pulmonary interstitial involvement is common in PM/DM patients. Approximately $75 \%$ of patients have subclinical interstitial pneumopathy, also associated with modified CT scans (10). A subset of PM/ DM have the "antisynthetase syndrome" characterized by myositis, ILD, inflammatory arthritis, "mechanic's hand" and Raynaud phenomenon along with a confirmation of circulating antitransfer RNA (anti-tRNA) synthetase autoantibody (e.g., antiJo-1). Often times the PM/DM-ILD is associated with the presence of one of the myositis-specific antibodies, with anti-Jo-1 having the strongest association with ILD followed by other not as commonly assessed tRNA synthetase autoantibodies (e.g., antiPL-7 and anti-PL-12) (16). The 5-year survival rates for patients with PM/DM and ILD are similar to that reported in idiopathic NSIP, $60 \%$ for both. The fulminant onset usually associates an extensive NSPI associated with OP and should direct the clinician to look for an antisyntetasic syndrome or amyopathic dermatomyositis (associated with Anti MDA5 antibodies) with increased mortality (10). Case reports and case series show favorable responses to immunosuppressive regimens. Typical agents used to treat PM/DM-ILD include corticosteroids along with CYC, cyclosporine, tacrolimus, AZA, or MMF. Recent case series also suggest that treatment with intravenous immune globulin or rituximab may be useful in refractory cases (17-19).
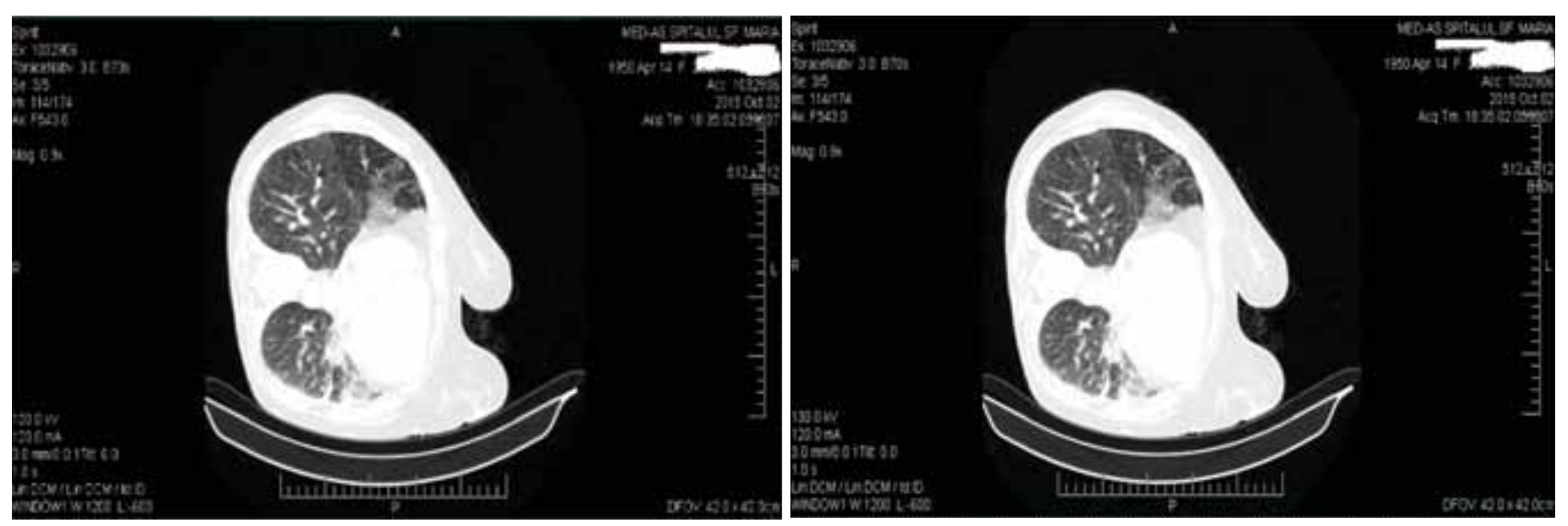

FIGURE 2. Ground glass pattern to a patient with CREST syndrome 
We present the case of a 59-year-old (V.I.) patient diagnosed with idiopathic polymyositis of the adult (anti Jo1 positive) in june 2016, with pulmonary damage (interstitial pulmonary fibrosis with moderate restrictive syndrome and moderately low DLCO - 44\%), with lymphocytic alveolitis (CD8 + lymphocyte predominant on bronhoalveolar lavage), who received high doses of corticosteroids for approximately 9 months for lung damage until it was framed in the context of collagenosis. From the onset until the moment of diagnosis, clinical and functional worsening is observed, associating effortless desaturation hypoxemia with the relatively stable spirometric and alveolocapillary diffusion parameters, which is why immunosuppressive therapy was started. Cyclophosphamide treatment is instituted in pulstheraphy, clinical and functional improvement is observed at 4 months, pneumological evaluation with CT scans revealing groud glass images and reticulo-micronodular pattern mostly peribronchial and subpleural, also bilateral bronchiectasis, stationary appearance (Fig. 3).

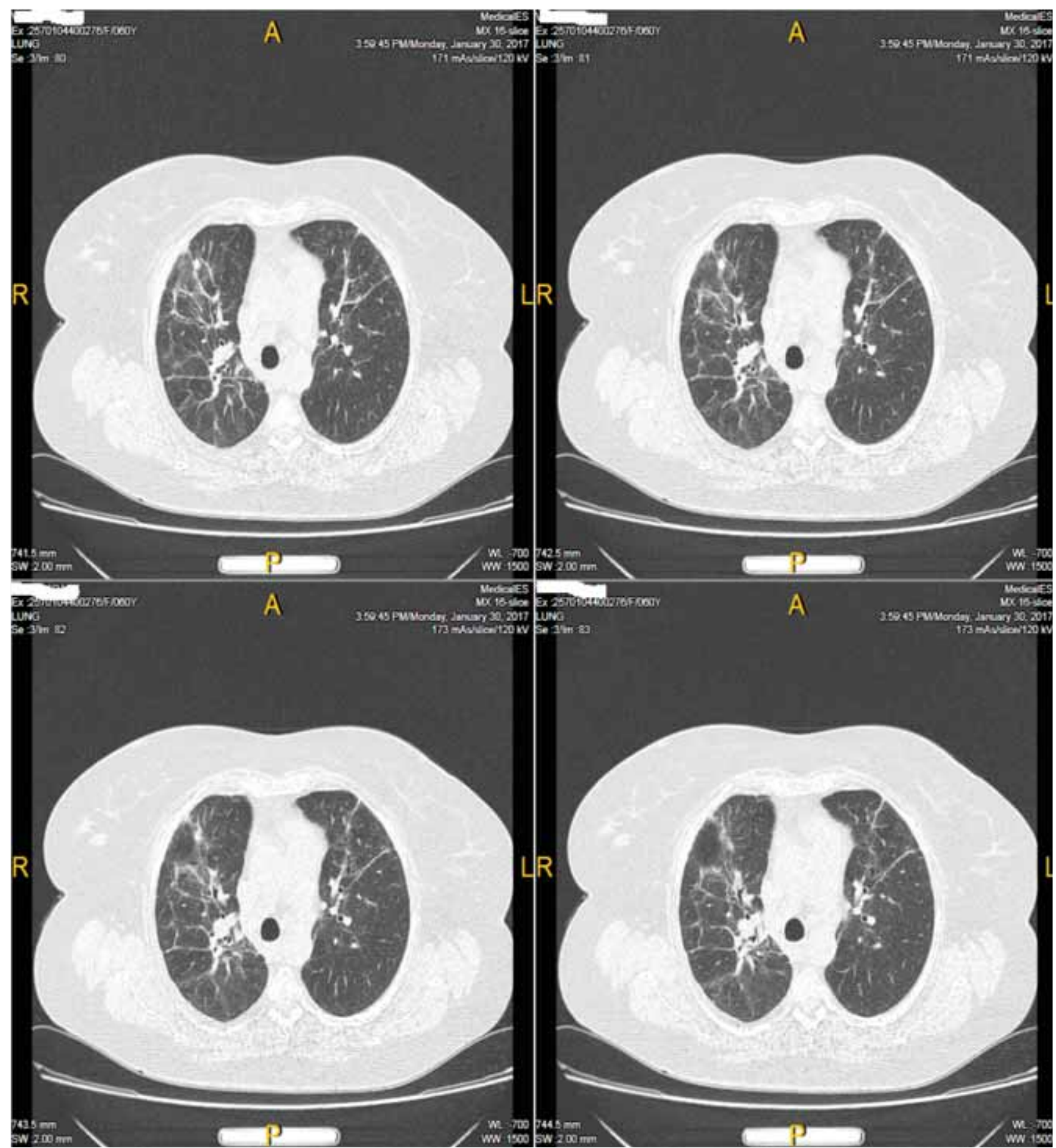

FIGURE 3. Mixed pattern of ground glass and peribronchial/subpleural reticulo-micronodular pattern, bilateral bronchiectasis in a patient with idiopathic polymyositis of the adult 
In conclusion, interstitial diffuse pneumopathy evolving towards pulmonary fibrosis in patients with connective tissue disease is associated with increased morbidity and mortality. High-resolution computed tomography is the gold standard in the early diagnosis of lung damage in these patients, the

\section{REFERENCES}

1. Castelino Flavia V. Interstitial lung disease in connective tissue diseases: evolving concepts of pathogenesis and management. Arthritis Res Ther. 2010; 12(4): 213.

2. Castelino F.V., Goldberg H., Dellaripa P.F. American College of Rheumatology Annual Meeting. San Francisco, CA; 2008. The impact of rheumatologic evaluation on the management of patients with interstitial lung disease (ILD) [abstract 637].

3. Cooper G.S., Dooley M.A., Treadwell E.L., St Clair E.W., Parks C.G., Gilkeson G.S. Hormonal, environmental, and infectious risk factors for developing systemic lupus erythematosus. Arthritis Rheum. 1998 Oct. 41(10):1714-24.

4. Lettieri C.J., Veerappan G.R., Parker J.M. et al. Discordance between general and pulmonary pathologists in the diagnosis of interstitial lung disease. Respir Med. 2005 Nov. 99(11):1425-30.

5. Fischer A., du Bois R. Interstitial lung disease in connective tissue disorders. Lancet 2012; 380: 689-698.

6. High-Resolution Computed Tomography in the Diagnosis of Diffuse Lung Disease: A Clinical Perspective. Wells, Athol U. Semin Respir Crit Care Med. 2003; 24(4),

7. Flaherty K.R., Toews G.B., Travis W.D. et al. Clinical significance of histological classification of idiopathic interstitial pneumonia. Eur Respir J. 2002; 19(2):275-283.

8. Bouros D., Wells A.U., Nicholson A.G. et al. Histopathologic subsets of fibrosing alveolitis in patients with systemic sclerosis andtheir relationship to outcome. Am J Respir Crit Care Med. 2002; 165(12):1581-1586.

9. Gabbay E., Tarala R., Will R. et al. Interstitial lung disease in recent onset rheumatoid arthritis. Am J Respir Crit Care Med 1997; 156(2 pt 1):528-535.

10. Solomon et al. Connective Tissue Disease-Associated Interstitial Lung Disease: A Focused Review. Journal of Intensive Care Medicine 2013 vol XX(X) 1-9.

11. Kim E.J., Collard H.R., King T.E. Jr. Rheumatoid arthritisassociated interstitial lung disease: the relevance of histopathologic and radiographic pattern. Chest. 2009; 136(5):1397-1405. pattern described being predictive of treatment response and disease progression. Treatment should be initiated as soon as possible, sometimes in an aggressive manner and interdisciplinary collaboration is essential.

Conflict of interest: none declared Financial support: none declared

12. Tsuchiya Y., Takayanagi N., Sugiura H. et al. Lung diseases directly associated with rheumatoid arthritis and their relation to outcome. Eur Respir J. 2011; 37(6):1411-1417.

13. Baruch D. Jakubovic, Andrea Donovan, Peter M. Webster, Neil H. Shear. Methotrexate-induced pulmonary toxicity Can Respir J. 2013 May-Jun and 153-155, 20(3).

14. Schurawitzki H., Stiglbauer R., Graninger W. et al. Interstitial lung disease in progressive systemic sclerosis: high-resolution CT versus radiography. Radiology. 1990; 176(3):755-759.

15. Moore O.A., Goh N., Corte T. Rouse H., Hennessy O., Thakkar V. et al. Extent of disease on high-resolution computed tomography lung is a predictor of decline and mortality in systemic sclerosis-related interstitial lung disease. Rheumatology (Oxford). 2013; 52.

16. Simeon-Aznar C.P. et al. Effect of mycophenolate sodium in scleroderma-related interstitial lung disease. Clin Rheumatol. 2011; 30(11):1393-1398

17. Fathi M., Dastmalchi M., Rasmussen E., Lundberg I.E., Tornling G. Interstitial lung disease, a common manifestation of newly diagnosed polymyositis and dermatomyositis. Ann Rheum Dis. 2004; 63(3):297-301.

18. Marie I., Hachulla E., Cherin P. et al. Interstitial lung disease in polymyositis and dermatomyositis. Arthritis Rheum. 2002; 47(6):614-622.

19. Suzuki Y., Hayakawa H., Miwa S. et al. Intravenous immunoglobulin therapy for refractory interstitial lung disease associated with polymyositis/dermatomyositis. Lung. 2009; 187(3):201-206.

20. Ball E.M., Savage E.M., Pendleton A. Refractory anti-synthetase syndrome treated with rituximab. Rheumatology (Oxford). 2010; 49(5):1013. 